\title{
Acceleration of Solar Energetic Particles by the Shock of Interplanetary Coronal Mass Ejection
}

\author{
Shanwlee Sow Mondal ${ }^{1,2}$ (i) , Aveek Sarkar $^{1}$ (D), Bhargav Vaidya ${ }^{3}$ (D) , and Andrea Mignone ${ }^{4}$ (10 \\ ${ }^{1}$ Astronomy and Astrophysics Division, Physical Research Laboratory, Ahmedabad 380009, India; shanwlee@prl.res.in, shanwlee.sowmondal@gmail.com \\ ${ }^{2}$ Indian Institute of Technology, Gandhinagar, Gujarat 382355, India \\ ${ }^{3}$ Discipline of Astronomy, Astrophysics and Space Engineering, Indian Institute of Technology Indore, Simrol, Indore 453552, India \\ ${ }^{4}$ Dipartimento di Fisica, Universita di Torino, via P. Giuria 1, I-10125 Torino, Italy \\ Received 2021 April 23; revised 2021 October 1; accepted 2021 October 1; published 2021 December 13
}

\begin{abstract}
Interplanetary coronal mass ejection (ICME) shocks are known to accelerate particles and contribute significantly to solar energetic particle events. We have performed magnetohydrodynamic-particle in cell simulations of ICME shocks to understand the acceleration mechanism. These shocks vary in Alfvénic Mach numbers as well as in magnetic field orientations (parallel and quasi-perpendicular). We find that diffusive shock acceleration plays a significant role in accelerating particles in a parallel ICME shock. In contrast, shock drift acceleration (SDA) plays a pivotal role in a quasi-perpendicular shock. High-Mach shocks are seen to accelerate particles more efficiently. Our simulations suggest that background turbulence and local particle velocity distribution around the shock can indirectly hint at the acceleration mechanism. Our results also point toward a few possible in situ observations that could validate our understanding of the topic.
\end{abstract}

Unified Astronomy Thesaurus concepts: Solar energetic particles (1491); Solar coronal mass ejections (310)

Supporting material: animations

\section{Introduction}

Solar energetic particles (SEPs) are nonthermal particles emanating from the Sun. Their energy can be several orders of magnitude higher than the ambient solar wind (SW) particles. These particles can be electrons, protons, or heavy ions. Usually, SEPs are detected soon after solar flares or when the heliospheric particle detectors encounter interplanetary coronal mass ejections (ICMEs). They have been detected at different heliospheric distances by multiple in situ spacecraft. SEPs can achieve energies up to several hundred $\mathrm{MeV}$ (Reames 1999) and sometimes even to $\mathrm{GeV}$ (Ryan et al. 2000). Intense SEP events may become hazardous for humans in space and can also damage the electronics of space instruments (Feynman \& Gabriel 2000).

These energetic events are considered to originate via two different mechanisms-particle acceleration in CME-driven shock waves (gradual SEP) and magnetic reconnection (impulsive SEP; Reames 1999). Impulsive SEP events occur for a shorter duration ( $\leqslant$ one day), with an occurrence frequency of about 1000 per year during solar maxima (Reames 2002). These are of low intensities. Gradual SEP events continue for a longer duration (several days). They are relatively rare (a few tens per year; Reames 2002) and produce proton flux several orders of magnitude larger than impulsive SEP events (Klein \& Dalla 2017). It is not easy to distinguish between these two processes in practice as they are intertwined and may co-occur. Magnetic reconnection may happen at the current sheet located behind the CME and at regions where the CME interacts with the ambient SW or fragmented current sheets of the posteruptive environment. Shock acceleration may occur in the region where the reconnection jet interacts with the ambient coronal material and produces an outflow termination shock and shock waves driven by the super magnetosonic motion of the CME (Klein \& Dalla 2017).
Multiple in situ observations have demonstrated (e.g., Sheeley et al. 1983; Gopalswamy 2003; Kahler 2004) the existence of SEPs during the passage of ICMEs. It is believed that the SW particles interacting with the shocks ahead of the ICMEs may get energized to such high energies (Lee \& Fisk 1982).

SEPs' peak intensities are found to be correlated with the speed and other physical parameters of the associated CMEs (Kahler 2001; Kahler \& Vourlidas 2005, 2013; Desai \& Giacalone 2016; Kouloumvakos et al. 2019). The strength of ICME-driven shock waves is usually determined by the Alfvénic Mach number $\left(M_{A}=u / v_{A}\right)$, where $v_{A}=B / \sqrt{4 \pi \rho}$ is the local SW Alfvén speed, and $u$ is the upstream SW speed in the shock rest frame. Commonly observed ICME-driven shocks possess $M_{A} \approx 2-4$ (Berdichevsky et al. 2000; Oh et al. 2007). However, for stronger ICMEs, $M_{A}$ can go beyond 5 (Lugaz et al. 2015). A powerful shock was recorded (Russell et al. 2013) on 2012 July 23, by the STEREO-A spacecraft, where the Alfvénic Mach number was determined to be $\approx 21$ (Riley et al. 2016). Proton energy spectra obtained from the particle detectors on board STEREO-A have shown that particles are energized up to $100 \mathrm{MeV}$ (Russell et al. 2013).

Like other astrophysical shocks, ICME shocks are also collisionless in nature. The particle mean-free path of the system is much larger than the shock length scale (say, the width of the shock) or gyroradii of the particles. Primarily two mechanisms are thought to be responsible for particle acceleration in these kinds of systems-diffusive shock acceleration (DSA; Fermi 1954; Krymskii 1977; Bell 1978; Blandford \& Ostriker 1978) and shock drift acceleration (SDA; Armstrong et al. 1985; Decker 1988). Depending on the upstream magnetic field's orientation with respect to the shock normal, the respective mechanism dominates. For example, in the case of quasi-parallel shocks, where the ambient magnetic field makes an angle in the range $0^{\circ}<\theta<45^{\circ}$ with the shock normal, DSA works as the dominant mechanism in accelerating 
the charged particles. Particles get scattered by magnetic turbulence and undergo repetitive head-on interactions with upstream and downstream plasma across the shock, thereby gaining energy. DSA is considered to be the most efficient mechanism responsible for the origin of highest-energy particles, mostly observed at quasi-parallel shocks. For strong $\left(M_{A} \gg 1\right)$ nonrelativistic quasi-parallel shocks, the energy spectra of DSA maintains a power law, $f(E) \propto E^{-1.5}$ (Caprioli \& Spitkovsky 2014a). DSA is considered to be the promising theory behind the genesis of gradual SEP events.

On the other hand, in quasi-perpendicular shocks, where the angle between the upstream magnetic field and the shock normal lies in the range $45^{\circ}<\theta<90^{\circ}$, SDA plays a pivotal role in particle acceleration. In this case particles gain energy by drifting along the shock in the direction of the induced electric field (Decker 1983; Reames 2012).

Particle acceleration is common in other astrophysical shocks such as in supernova remnants (Helder et al. 2012) or in extragalactic jets (Romero et al. 2017). These shocks are considered to be the primary sources of galactic cosmic rays (CRs). Many efforts have already been made to understand the origin and transport of high-energy particles (CRs), their complex nonlinear interactions with the background thermal plasma, and the ambient magnetic field (Fermi 1949, 1954; Blandford \& Ostriker 1978).

The most obvious framework to study the evolution of CRs numerically around a shock would be the particle in cell (PIC) method. In the PIC, electrons and ions are treated as particles, and are therefore suitable to study plasma kinetics in the presence of a background electromagnetic field (Spitkovsky 2005). However, one needs to resolve the electron skin depth in the PIC code, which makes the simultaneous study of shock evolution and particle acceleration highly expensive.

Recently the field has been enriched with the development of hybrid-PIC codes where the electrons are considered as part of the background thermal fluid, and ions are treated as kinetic particles (Lipatov 2002; Gargaté et al. 2007). Such simulations need to resolve the ion-skin depth of the medium, making the simulations computationally expensive. With the availability of powerful machines, numerous works have been done where simulations were performed (Caprioli \& Spitkovsky 2013, 2014a, 2014b, 2014c) with relatively large domains and for long durations, but still, only the initial stages of shock evolution and particle acceleration have been captured. However, the transition of charged particles from nonrelativistic to relativistic regimes and the fraction of ions participating in the process would only be known when we can study the long-term evolution of collisionless shocks. The magnetohydrodynamic-particle in cell (MHD-PIC) approach is more relevant for such purposes (Bai et al. 2015; Mignone et al. 2018; van Marle et al. 2018). This method can describe the nonlinear interaction between thermal (ions+electrons) and nonthermal energetic particles. The method conveniently ignores microscopic plasma scales but captures the kinetic effect of ions by resolving the particles' gyroradii.

The MHD formulations carry out the evolution of the thermal plasma consisting of both electrons and ions, and the dynamics of the particles (nonthermal ions) are studied using the PIC method. All the nonthermal particles are extracted from the thermal fluid itself. After extracting a certain fraction of ions from the background plasma, the Lorentz force experienced by these charged particles in the background fluid's electric and magnetic fields is calculated. This force now acts as the feedback force that the particles exert on the background fluid and thus modifies its evolution. Recent usage of adaptive mesh refinement on the background thermal plasma (van Marle et al. 2018) has made the MHD-PIC a more computationally affordable method.

A numerical study of particle acceleration in heliospheric shocks was initiated using the Monte Carlo simulation (Baring et al. 1997). It showed that the particle energy spectra obtained from the simulation are in good agreement with the in situ energy spectra obtained from the interplanetary shock, suggesting an active DSA mechanism in the quasi-parallel heliospheric shock.

An extensive study of particle acceleration by CME shocks has been done using the particle acceleration and transport in heliosphere (PATH) code (Zank et al. 2000). This model includes spherically symmetric SW, in which a CME-driven shock wave propagates. Particles are injected at the shock using an injection model where the number of injected particles is a small fraction of the thermal SW particles. Injected particles get accelerated through DSA. Some of these particles also escape far upstream through diffusion. The outcome of such modeling includes the temporal evolution of energetic particle spectra and intensity profile at all the spatial locations (both upstream and downstream), and the determination of the particle injection energy and the maximum energy of particles accelerated at the shock.

The same PATH code was later modified to include shocks with arbitrary strengths (Rice et al. 2003), giving a better estimation of the maximum particle energy for each shock. The transport of energetic particles ( $\mathrm{Li}$ et al. 2003) escaping the shock, simulating the acceleration of heavy ions ( $\mathrm{Li}$ et al. 2005 b) also have been studied using the same. It has also been used (Zank et al. 2004, 2006) to study particle acceleration at a perpendicular interplanetary shock. Modeling individual SEP events using the PATH code has been pursued by several authors (Li et al. 2005a; Zank et al. 2007; Verkhoglyadova et al. 2009, 2010). Li et al. (2012) have studied the particle acceleration in oblique shock. Their results suggest that close to the Sun $(r<0.1 \mathrm{au})$, quasi-parallel shocks are better particle accelerators than quasi-perpendicular shocks.

A 2D extension of the original PATH code (Zank et al. 2000) was developed by $\mathrm{Hu}$ et al. (2017). This new model can take care of the evolution of the background SW and propagate the CME shock in a 2D domain. It also calculates the particle acceleration at the shock along with their diffusion and convection in the upstream and downstream regions. This 2D model can also capture the longitudinal distribution of the CME.

Using the hybrid-PIC code, dHybrid Gargaté et al. (2007) have studied the particle acceleration in ICME shocks. This kind of study can be categorized more as a local shock simulation. Instead of taking the whole longitudinal distribution of the shock, a particular region is considered for the simulation, and acceleration of nonthermal particles is studied. The simulation could successfully capture the detailed spatial and temporal information of the electromagnetic waves generated due to counter-streaming ions in the shock upstream. However, the hybrid method's high demand for computational resources restricts them to simulate only up to $15 \mathrm{~s}$ of the 
particle acceleration. The MHD-PIC method is a natural choice to deal with such difficulties.

The present work utilizes the MHD-PIC version of the PLUTO code (Mignone et al. 2018). Unlike the hybrid-PIC code, through this approach, one can track the shock for several minutes and evolve the energy spectrum of nonthermal particles. We simulate ICME shocks with various strengths (with different $M_{A}$ ) and magnetic configurations (parallel shock as well as perpendicular shock). The evolution of nonthermal protons is studied until their energy spectrum gets saturated. The aforementioned simulations explain the physical processes behind gradual SEP events.

The rest of the article is organized as follows. We describe the overall simulation setup in Section 2. Parallel and quasiperpendicular shocks with high Mach numbers are elaborated in Section 3. Results of low Mach shocks are detailed in Section 4. Finally, we summarize our results in Section 5.

\section{Simulation Setup}

A complete derivation of the MHD-PIC formalism is given in Bai et al. (2015). The MHD-PIC version of the PLUTO code is also explained in Mignone et al. (2018).

We have simulated ICME shocks of different Alfvénic Mach numbers and analyzed particle acceleration in each of them. ICME shocks observed at 1 au vary over a wide range of Alfvénic Mach numbers. A comparative study is performed to understand the role of the shocks' strength in the particle energization process. In all our simulations density, temperature, and magnetic field scales are taken to be $n_{0}=10 \mathrm{~cm}^{-3}$, $T_{0}=5.6 \times 10^{4} \mathrm{~K}$, and $B_{0}=2.817 \mathrm{nT}$. Our length, time, and velocity scales are chosen in terms of ion-skin depth $\left(c / \omega_{\mathrm{pi}}=72 \mathrm{~km}\right)$, inverse of ion cyclotron frequency $\left(\Omega^{-1}=3.69 \mathrm{~s}\right)$, and Alfvén velocity $\left(v_{A}=19.5 \mathrm{~km} \mathrm{~s}^{-1}\right)$. Here, $c$ is the speed of light and $\omega_{\mathrm{pi}}$ is the ion plasma frequency. To emulate the SW plasma at $1 \mathrm{au}$, simulation domains are initially filled with a plasma of uniform density $n_{\mathrm{bg}}=0.5 n_{0}\left(5 \mathrm{~cm}^{-3}\right)$, and temperature $9 T_{0}\left(5 \times 10^{5} \mathrm{~K}\right)$ with uniform magnetic field strength of $B_{\mathrm{bg}}=3.55 B_{0}(10 \mathrm{nT})$ whose orientation is different for parallel and quasi-perpendicular cases. Each simulation domain consists of a $2 \mathrm{D}$ rectangular box of length $L_{x}=1.5 \times 10^{5}\left(c / \omega_{\mathrm{pi}}\right)$ and $L_{y}=5 \times 10^{3}\left(c / \omega_{\mathrm{pi}}\right)$ with uniform resolution $\Delta x=\Delta y=10\left(c / \omega_{\mathrm{pi}}\right)$.

\subsection{Shock Generation}

Generally, in the inner heliosphere, ICMEs travel faster than the ambient SW, with both reaching supersonic and super Alfvénic speeds. A shock wave gets developed ahead of the ICME. In between the shock and the ICME, a turbulent region matures-known as the sheath region (Kilpua et al. 2017).

In numerical simulations such as the ones considered in this work, shocks are typically formed by using the piston method (Gargaté et al. 2014; Bai et al. 2015). A leftward super Alfvénic flow driven continuously from the right boundary gives rise to a rightward propagating shock after getting reflected from the left conductive wall. The shock's strength and speed are controlled by adjusting the velocity of the upstream flow. The top and bottom boundaries maintain the periodic boundary condition.

The method described above can simulate an ICME shock quite nicely. Using this method, we capture the shock and part of the sheath region of an ICME in the downstream reference frame. With other parameters chosen to emulate SW parameters at $1 \mathrm{au}$, the inflow velocity is identified with the relative velocity between the SW and the CME. For our purpose we have chosen shocks with strong compression ratio, $r=\frac{\rho_{d}}{\rho_{u}} \approx \frac{\gamma+1}{\gamma-1} \approx 4$, where $\gamma=5 / 3$ is the adiabatic index and $\rho_{u}$ and $\rho_{d}$ represent the upstream and downstream densities, respectively.

The shock converts the kinetic energy of the upstream flow to the thermal energy of the downstream plasma. As a result the downstream temperature is increased to $T_{d}=v_{u}^{2} /(r-1) \sim v_{u}^{2} / 3$ (in code units), where $v_{u}$ is the upstream velocity in the downstream reference frame. For a strong shock the upstream temperature $\left(T_{u}\right)$ is independent of the downstream temperature (Bai et al. 2015).

\subsection{Particle Injection}

Unlike the Hybrid-PIC, the present MHD-PIC is not capable of generating nonthermal particles self-consistently from the thermal plasma. Therefore a strategy has to be adopted to inject protons that participate in the acceleration process. Even though electrons do also get energized in reality, limited computational power prohibits one from simulating their dynamics. Therefore we assume all electrons are thermalized and do not participate at the energization process. Only protons are capable of participating in the nonthermal process. In this article, proton and particle both represent the same entity. The participating protons are injected throughout the simulation. Equivalent mass, momentum, and energy of protons are subtracted from the background to ensure conservation.

The amount of injected protons is chosen in accordance with Bai et al. (2015), inspired by Caprioli \& Spitkovsky (2014a). The fraction of the injected proton mass to the mass swept away by the shock, $\eta$ is chosen to be $3 \times 10^{-3}$ in all our production runs. We have checked that if we increase this fraction by a significant amount, the injected protons can disrupt the shock through backreaction. On the other hand, a too-small value of $\eta$ may not contribute to the backreaction, and the proton acceleration process may become inefficient. The location of the shock front is tracked every time using the PLUTO shock tracking algorithm (Mignone et al. 2018). Once the shock is detected, the amount of particles we inject is a fixed fraction, $\eta$ of the mass swept by the shock as mentioned above. The number of injected particles is equivalent to 10 particles per cell at background fluid density $n_{0}$.

Since the background thermal plasma and injected particles are treated differently in the MHD-PIC code, an ad hoc injection prescription needs to be adopted for proton injection. An injection recipe can be adopted by calibrating the MHDPIC simulation with a more self-consistent hybrid-PIC simulation. Nevertheless, in the absence of such results, so far monoenergetic injection recipes have been used in MHDPIC (Bai et al. 2015; Mignone et al. 2018; van Marle et al. 2018). However, to inject particles with a wide range of energies, we have primarily used a Maxwellian distribution with downstream characteristic temperature.

The following sections are dedicated to the numerical setup and results obtained from simulations of particle acceleration in different shocks. 


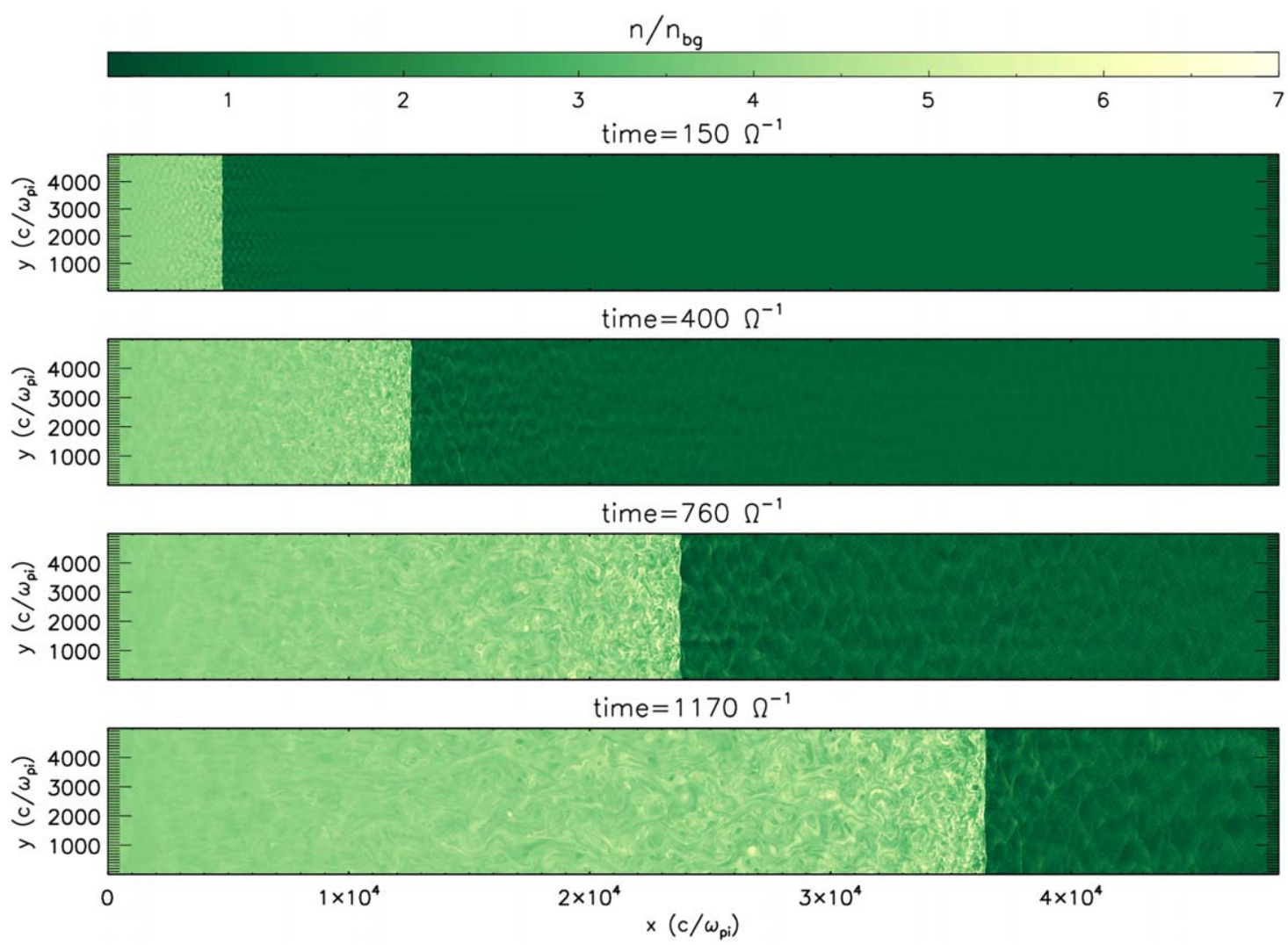

Figure 1. Density evolution of the parallel shock $\left(M_{A} \approx 19\right)$, moving toward the right. Only a fraction of the simulation domain is depicted here. (An animation of this figure is available in the online version. The animation captures the density evolution of the parallel shock $\left(M_{A} \approx 19\right)$, moving toward right from time $t=0$ to $1170 \Omega^{-1}$. Only a fraction of the simulation domain is depicted here).

(An animation of this figure is available.)

\section{Simulation of ICME Shock with $M_{A} \approx 19$}

\subsection{Numerical Setup}

Keeping in mind the detection of a strong ICME shock (Russell et al. 2013; Riley et al. 2016) at 1 au with Alfvénic Mach $\approx 19$ in the downstream reference frame, we initially simulate an ICME shock with the same Alfvénic Mach number. An initial background magnetic field of strength $B_{\mathrm{bg}}=3.55 B_{0}$ is applied along the $x$ direction, which is parallel to the shock normal. Number of particles injected at the shock front depends on $\eta$ times the amount of mass swept by the shock, with $\eta=3 \times 10^{-3}$ as mentioned in Section 2.2. In a system like this, an instability is expected to develop due to the current generated by the energetic protons escaping the shock front thereby perturbing the upstream magnetic field (Bell 2004). The present resolution is sufficient to resolve the most unstable mode of such instability. Near the shock front the wavelength of this mode is approximately $3 \pi\left(B_{\mathrm{bg}} / B_{0}\right) / \eta M_{A}=587\left(c / \omega_{\mathrm{pi}}\right)$ (Bai et al. 2015). Considering the SW and ICME speed to be $400 \mathrm{~km} \mathrm{~s}^{-1}$ and $2250 \mathrm{~km} \mathrm{~s}^{-1}$, respectively, we take their relative speed to be $-1850 \mathrm{~km} \mathrm{~s}^{-1}$. Inflow speed of $-94.87 v_{A}$ $\left(=-1850 \mathrm{~km} \mathrm{~s}^{-1}\right)$ produces a rightward propagating shock of Alfvénic Mach number $\approx 19$. The detailed generation mechanism of the shock is described in Section 2.1. Once the shock is formed, particles are injected following the prescription discussed in Section 2.2.

\subsection{Shock Propagation}

Density snapshots of the propagating shock are shown in Figure 1. Note that only a small part of the whole simulated domain is shown here. In the beginning, injected protons move freely in the upstream region. The motion of the energetic particles across the shock generates a current $\boldsymbol{J}$, primarily parallel to the ambient upstream magnetic field. The current initially perturbs the mean upstream magnetic field to generate Alfvén waves. Once the current gets stronger due to the high flux of energetic particles, it generates an instability known as Bell instability after its founder (Bell 2004). That, in turn, produces a perpendicular $\left(\delta \boldsymbol{B} \sim B_{\perp}\right)$ magnetic field in the upstream (Bell 2005; Reville \& Bell 2012). This magnetic field generates a force $(-\boldsymbol{J} \times \delta \boldsymbol{B})$, which acts on the local plasma and evacuates the region. As a result, low-density cavities are produced. Suprathermal particles occupy these cavities. Once the maximum current carrying particles' gyroradii become comparable to the cavities' size, the growth of the instability ceases (Caprioli \& Spitkovsky 2013). Freely moving protons in the region get scattered by the density cavities.

To estimate the density cavities' size we consider the average density power spectrum over a short time window $\left(t=1000-1170 \Omega^{-1}\right)$, toward the end of the simulation. The density power spectrum (Figure 2) of the shock upstream plasma is derived from a region $330\left(c / \omega_{\mathrm{pi}}\right)$ ahead of the shock front, having width $2400\left(c / \omega_{\mathrm{pi}}\right)$. Sufficient cavities have formed at this location. The peak of the power spectrum, which is at $55\left(c / \omega_{\mathrm{pi}}\right)$ indicates the dominant length scale of the cavities. The magnetic field at the same location averaged over 


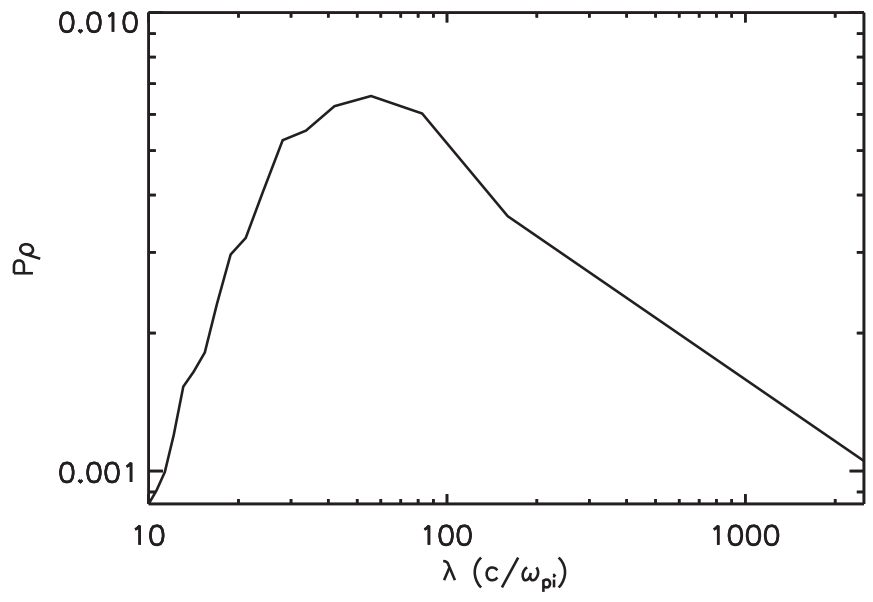

Figure 2. Fluctuating mass density $\left(\delta \rho / \rho_{0}\right)$ power spectrum during the nearsaturation phase of the simulation (averaged over time $t=1000-1170 \Omega^{-1}$ ). Here $\rho_{0}$ is the initial upstream density. The spectrum is derived at the location $330\left(c / \omega_{\mathrm{pi}}\right)$ ahead of the shock front (Figure 1), over a width $2400\left(c / \omega_{\mathrm{pi}}\right)$.

the horizontal and transverse directions is found to be $\approx 3.97 B_{0}$. Considering the typical particle velocity to be $\approx 232 v_{A}$ (local particle velocity distribution peaks around this velocity), we find the particle-gyroradius to be $\approx 58\left(c / \omega_{\mathrm{pi}}\right)$. The similarity in gyroradius and cavity size indicates the instability is saturated.

\subsubsection{Role of Instabilities in Shock Upstream}

Figure 3 shows the magnetic field evolution close to the shock front. For every time snap, the shock front is recentered

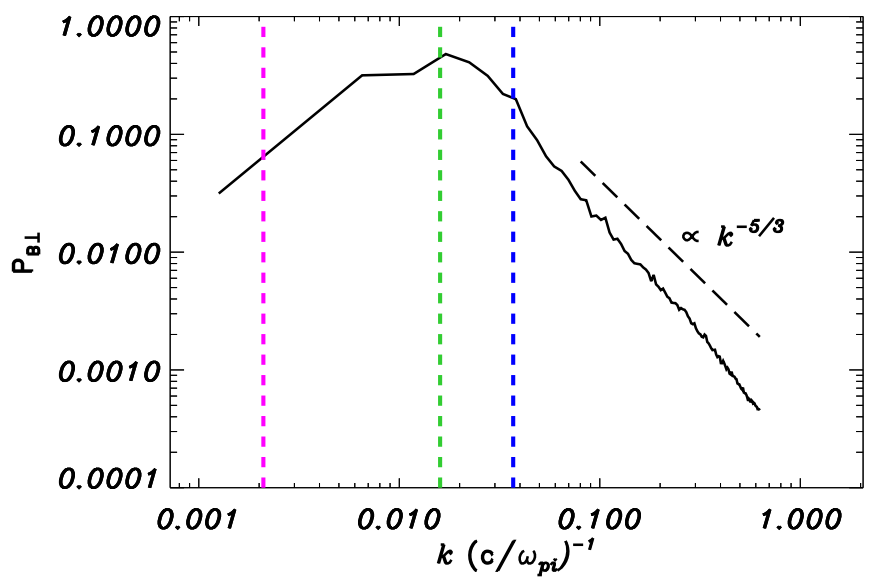

Figure 4. Dimensionless transverse magnetic energy power spectrum at $t=1170 \Omega^{-1}$ is calculated in a region of width $\approx 2400\left(c / \omega_{\mathrm{pi}}\right)$ just ahead of the shock. Blue and magenta dashed lines indicate the wave modes in resonance with the particles having energies $E_{\mathrm{sh}}$ and $300 \mathrm{E}_{\mathrm{sh}}$, respectively. The theoretical prediction of the fastest growing mode of the Bell's instability is marked with the green dashed line, calculated with the transversely averaged current at the shock front. The black dashed line indicates the Kolmogorov power-law slope.

at $x=0$. It is seen that in the shock precursor, along with the density, the magnetic field also becomes turbulent. The power spectrum of the perpendicular magnetic field, depicted in Figure 4 , describes the nature of the magnetic turbulence. The maximum wavenumber $\left(k_{\max }\right)$ is determined by the simulation resolution, whereas the minimum wavenumber $\left(k_{\min }\right)$ is set by the largest length scale of the chosen region. Alfvén waves,

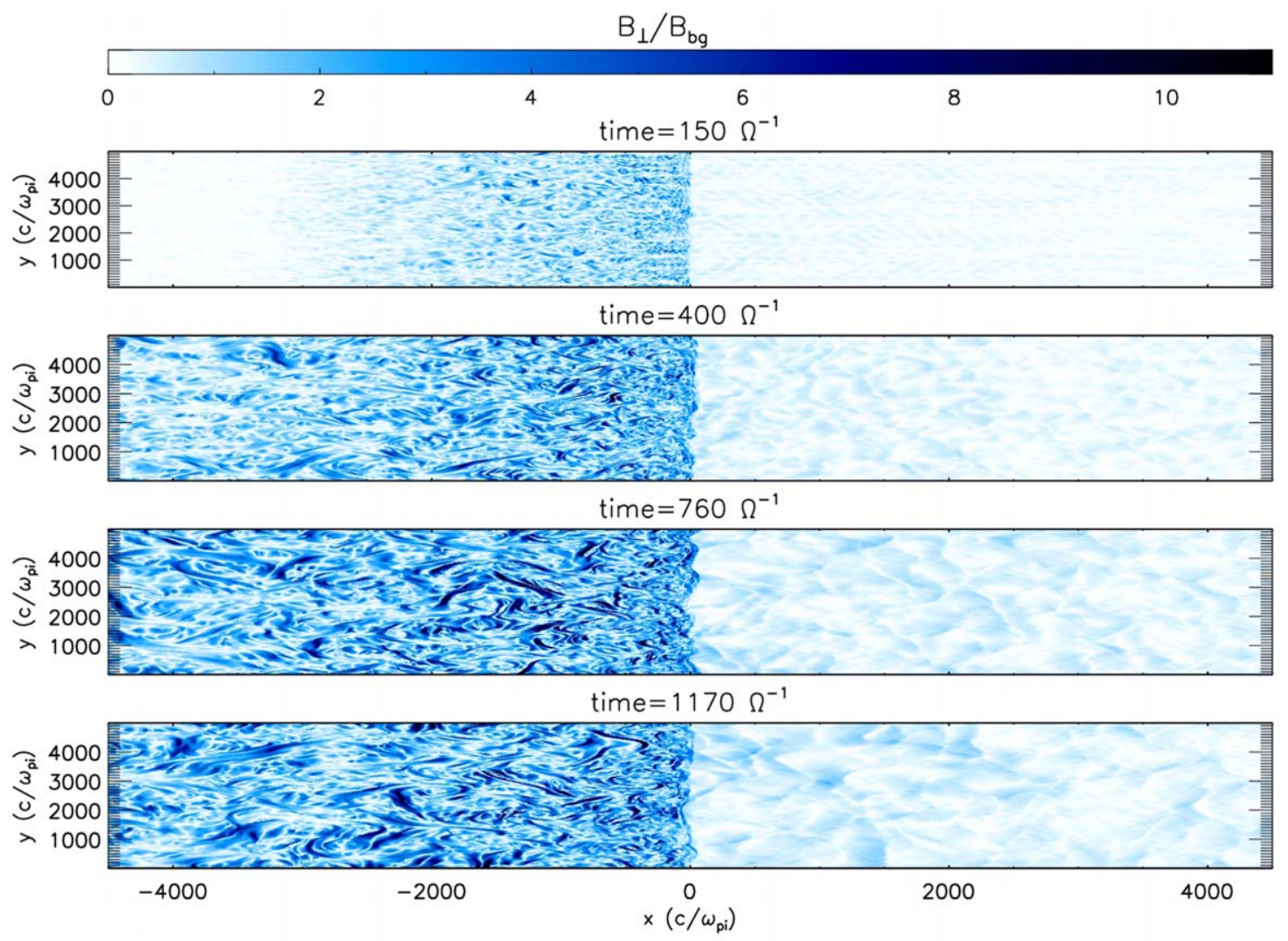

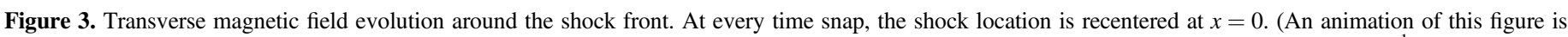

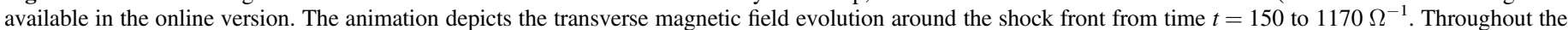
animation, the shock location is recentered at $x=0$ ).

(An animation of this figure is available.) 


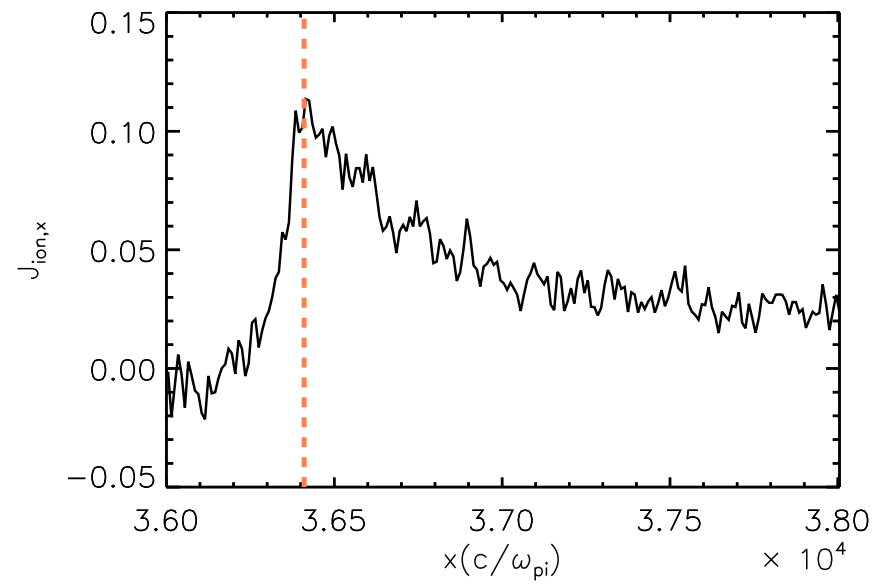

Figure 5. Transversely averaged $x$-component of the current density $\left(J_{\text {ion } x}\right)$ in normalized units. The dashed line indicates the location of the shock and the corresponding current is considered to calculate the theoretical value of the most unstable Bell mode (Figure 4).

initiated by the energetic particles in the shock upstream is natural to expect (Bell 2004). These waves, resonating with the gyrofrequency of the streaming energetic particles, generate an instability in the system known as resonant streaming instability (Bell 2004; Amato \& Blasi 2009). The mode of such instability achieves maximum growth rate at a wavenumber $k$ satisfying $k r_{g}\left(E, B_{\mathrm{bg}}\right)=1$, where $E$ is the energy of the particle gyrating with gyroradius $r_{g}$ in the background magnetic field of strength $B_{\mathrm{bg}}$ (Caprioli \& Spitkovsky 2014b). We find that in the region the longest (for particles with $E=E_{\mathrm{sh}}$ ) and shortest (particles with $E=300 E_{\mathrm{sh}}$ ) wavenumbers of the resonant mode are $3.74 \times 10^{-2}\left(\frac{c}{\omega_{\mathrm{pi}}}\right)^{-1}$ and $2.13 \times 10^{-3}\left(\frac{c}{\omega_{\mathrm{pi}}}\right)^{-1}$, respectively. While these two modes encompass the peak of the power spectrum (Figure 4), the wave mode corresponding to the spectral peak resonates with the gyrofrequency of the particles having energy $E=3 E_{\mathrm{sh}}$. However, the nonresonant hybrid (NRH) instability introduced by Bell (2004) also may exist in this atmosphere. The theoretical estimation of the fastest growing mode of this instability is found to be $k_{\mathrm{Bell}}=\frac{J_{\mathrm{ion}, x}}{2 B_{\mathrm{bg}}}$, where $J_{\mathrm{ion}, x}$ corresponds to the transversely averaged horizontal component of the current, generated due to the motion of the nonthermal protons, shown in Figure 5. Considering the upstream shock vicinity value of $J_{\text {ion, } x}$ and the initial background magnetic field $B_{\mathrm{bg}}$, we estimate the fastest growing mode of the Bell instability to be $k_{\text {Bell }}=1.59 \times 10^{-2}\left(\frac{c}{\omega_{\mathrm{pi}}}\right)^{-1}$, which nearly coincides with the wave mode corresponding to the peak of the transverse magnetic power spectrum. Among the two modes the nonresonant mode dominates when $\left(\frac{J_{\text {ion, } x} r_{g}}{B_{\mathrm{bg}}}\right) \gg 1$ (Bell 2004; Amato \& Blasi 2009). Using the local values of $J_{\text {ion, } x}, r_{g}$, and $B_{\mathrm{bg}}$ of the immediate shock upstream region, one finds the value of this nondimensional quantity to be $\approx 1.5$, which favors the NRH instability in the region. Following the peak, the magnetic field power spectrum falls off with the characteristic Kolmogorov slope $\left(k^{-5 / 3}\right)$.

Figure 6 shows the magnetic field map in a region close to the shock front. Instabilities described above generate magnetic fluctuations enhancing the local magnetic field by as much as twice the initial value at the shock precursor. Following the Rankine-Hugoniot shock condition, it is expected that the transverse magnetic components would get further amplified by a factor of 4 . In other words, the already preamplified magnetic field of the upstream would be amplified further up to 8 times with respect to the background due to shock compression, and propagate into the downstream region. However, the total magnetic field contour in Figure 6 shows an amplification by a factor of up to 15 at some locations of downstream. Clearly, a different magnetic field amplification mechanism is at play here. The initial shock surface gets perturbed by the upstream density disturbance and gets corrugated. At the corrugated surface, because of the nonaligned pressure and density gradient, vorticity gets introduced and drives the RichtmeyerMechkov Instability (RMI; Brouillette 2002). The turbulence introduced by the RMI helps to enhance the magnetic field further by stretching the field lines (Sano et al. 2012), indicating the possible activation of turbulent dynamo behind the shock. The role of the turbulent dynamo in the shock downstream has also been reported by earlier authors (e.g., Giacalone \& Jokipii 2007; Mizuno et al. 2011).

\subsubsection{Magnetic Field Enhancement in the Shock Downstream}

For further investigation in the turbulence saturation phase, the kinetic and magnetic energy power spectrum of the region is plotted in Figure 7. In the downstream region, 388 $\left(c / \omega_{\text {pi }}\right)$ away from the shock front, a strip of width $800\left(c / \omega_{\text {pi }}\right)$ is considered to calculate the power spectrum. For the kinetic energy spectrum the parameter $\rho^{1 / 2}[\boldsymbol{u}-\overline{\boldsymbol{u}}]$ is employed (Podesta et al. 2007), whereas for the magnetic energy spectrum $\boldsymbol{B}-\overline{\boldsymbol{B}}$ is considered. Here $\rho, \boldsymbol{u}, \boldsymbol{B}, \overline{\boldsymbol{u}}$, and $\overline{\boldsymbol{B}}$ are mass density, velocity, magnetic field, mean velocity, and mean magnetic field of the region respectively. To calculate the mean fields $(\overline{\boldsymbol{u}}, \overline{\boldsymbol{B}})$, a time window of about $347 \Omega^{-1}$ is considered. Higher magnetic energy toward the small scale is an indicator of a possible small scale active dynamo in the region.

\subsubsection{Particle Energization}

Turbulence in the shock upstream and downstream can scatter particles across the shock and energize them. As mentioned earlier, we inject Maxwellian distribution of particles with characteristic downstream temperature. A 2D particle energy spectrum at the evolved state of the simulation is shown in Figure 8. This figure depicts the particles' energy distribution around the shock. It clearly shows the existence of escaped high-energy particles in the shock upstream.

Figure 9 displays the maximum energy attained by the protons over time. We ran our simulation until maximum energy curve reached its plateau. Figure 10 depicts the evolution of the $1 \mathrm{D}$ energy spectrum $\left(E^{3 / 2}\right.$ compensated $)$ of the particles. These spectra are extracted from a region of width $1000\left(c / \omega_{\text {pi }}\right)$ just behind the shock. During the initial phase (e.g., $50 \Omega^{-1}$ ), the spectrum almost follows the injected Maxwellian distribution, and eventually particles gain energy over time. During the near-saturation phase, particles are seen to have gained energy up to $E=500 E_{\mathrm{sh}}(\approx 10 \mathrm{MeV})$ in the downstream region just behind the shock. The major part of the evolved spectrum follows $-3 / 2$ slope, indicating the active presence of the DSA mechanism.

The maximum energy of the SEPs during CME shocks, however, has been seen to have reached $100 \mathrm{MeV}$ (Russell 


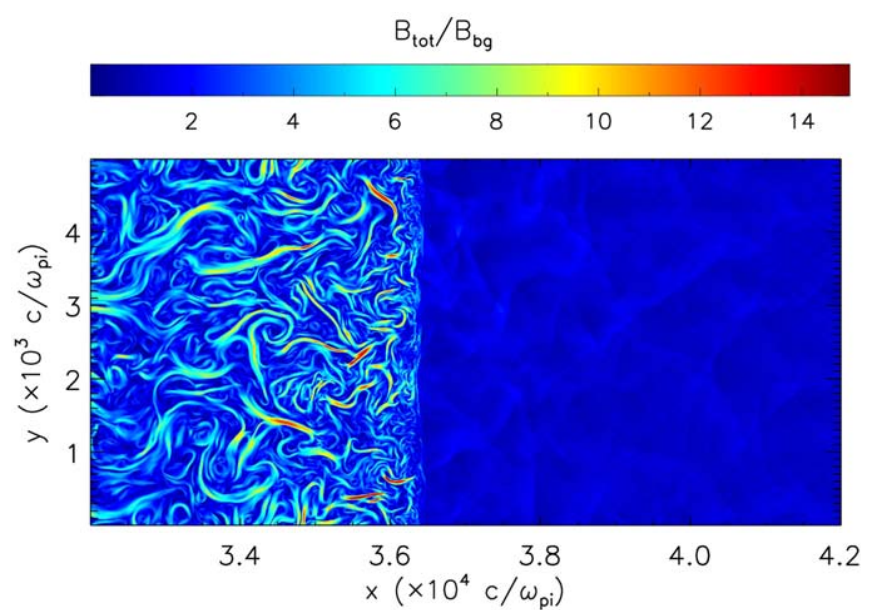

Figure 6. Total magnetic field contour close to the shock front at $t=1170 \Omega^{-1}$.

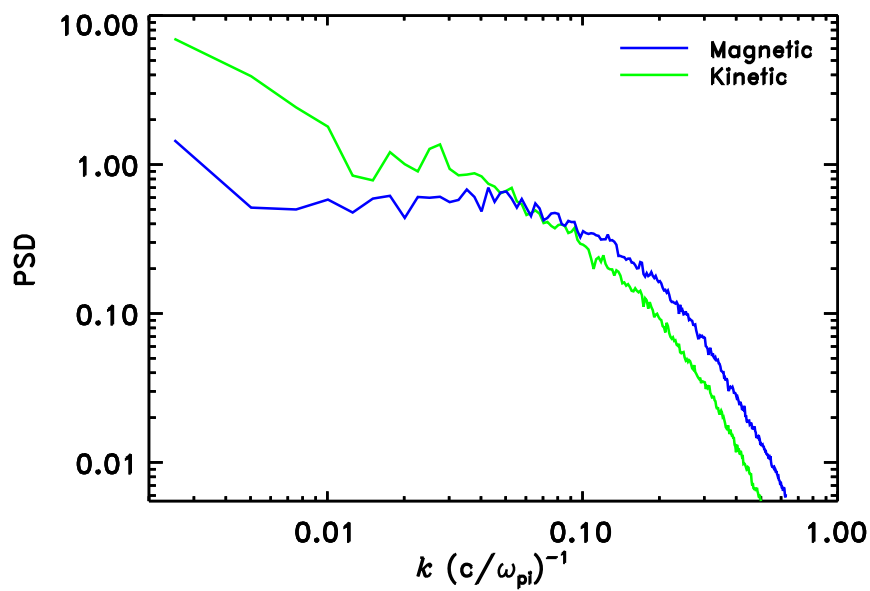

Figure 7. Dimensionless kinetic and magnetic energy power spectrum at $t=1170 \Omega^{-1}$ in a region of width $8000\left(c / \omega_{\mathrm{pi}}\right)$ in the downstream region 400 $\left(c / \omega_{\mathrm{pi}}\right)$ away from the shock front.

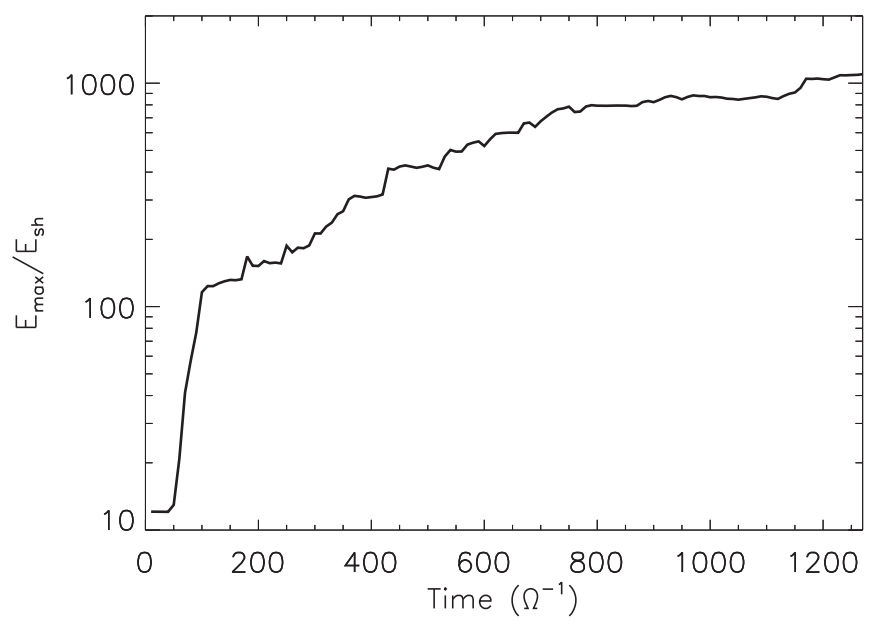

Figure 9. Time evolution of the maximum energy of the particles (normalized with respect to $\left.E_{\mathrm{sh}}\right)$ present in the entire domain. The curve plateaus at large times suggesting near-saturation.

et al. 2013). A possible reason behind not being able to produce such highly energetic protons in our simulation could be the size of the simulation box, which restricts the longer wave modes. Figure 11 compares the energy spectra for three different simulations with varying transverse box sizes, 250, 500 , and $5000\left(c / \omega_{\mathrm{pi}}\right)$, during their near-saturation phase. The tail of the protons' energy spectrum extends toward the higher energy with an increase in transverse box size, thereby indicating the role of the same in constraining the maximum energy gained by the protons.

In reality, SW demonstrates characteristic fluctuations. The ambient SW of our present simulation is initiated without any turbulent fluctuation. We envisage that introduction of such turbulent fluctuation in the initial atmosphere may play an important role in the particle energization process.

We have also compared our Maxwellian injection recipe with the monoenergetic injection recipe, where $E_{\mathrm{inj}}=10 E_{\mathrm{sh}}$,
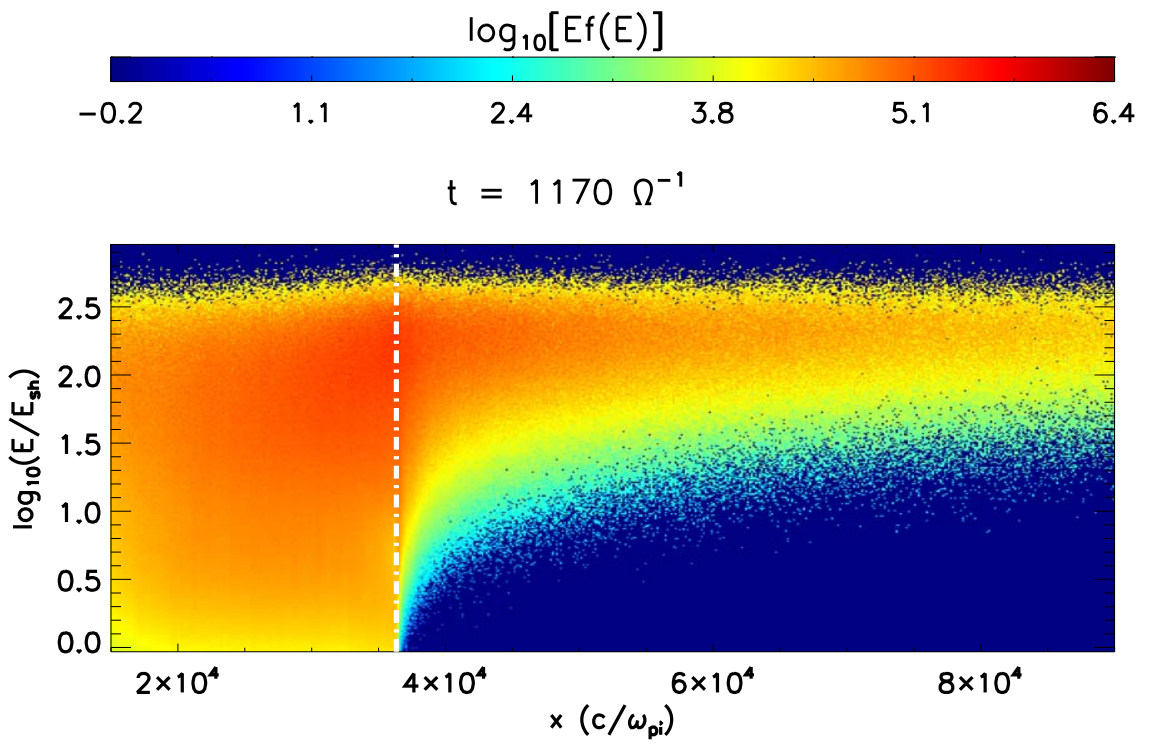

Figure 8. $2 \mathrm{D}$ energy spectrum, showing the particle energy distribution as a function of position $x$. Here $E_{\mathrm{sh}}$ is the shock energy given by $E_{\mathrm{sh}}=v_{u}^{2} / 2$ where $v_{u}$ is the upstream plasma speed in the downstream reference frame. Location of the shock front is indicated by the white dashed line. High-energy particles outrunning the shock are evident from this $2 \mathrm{D}$ spectrum confirming the commencement of particle acceleration. 


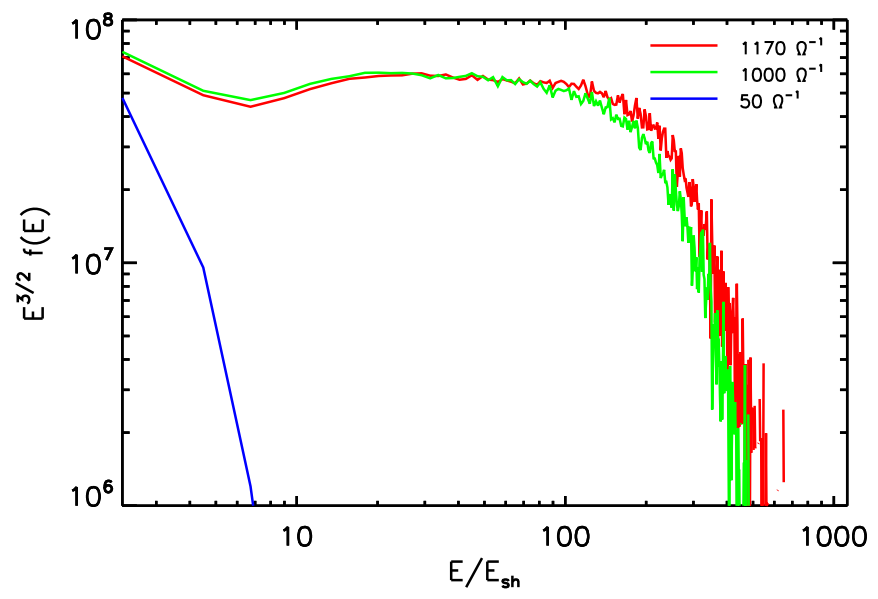

Figure 10. Evolution of downstream particle energy spectra $\left(E^{3 / 2}\right.$ compensated), extracted from a region of width $1000\left(c / \omega_{\mathrm{pi}}\right)$, just behind the shock. Flattening of the spectra ensures DSA is an efficient mechanism in particle acceleration.

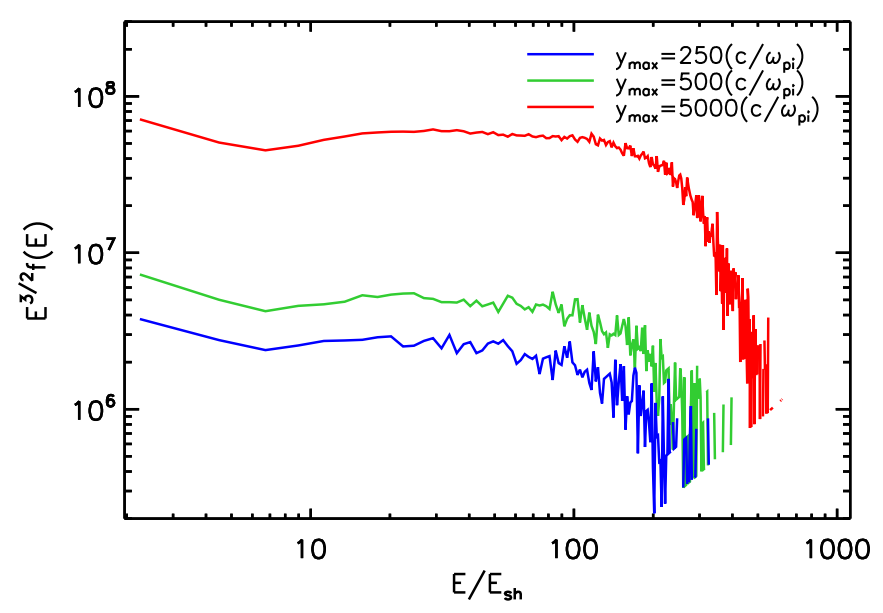

Figure 11. Saturated particle energy spectra for three different transverse domain sizes. Particle energization is seen to increase with the domain size.

typically employed by previous investigators (Bai et al. 2015; Mignone et al. 2018). In Section 2.2, we have already mentioned that in our present simulation, we choose to have a Maxwellian injection with downstream plasma temperature. For this case we had carefully chosen the combination of $\eta=3 \times 10^{-3}$ and the number of particles per cell to be 10 , so that the particles do not disrupt the shock. On the other hand, the monoenergetic injection with the above combination of $\eta$ and the number of particles per cell disrupts the shock indicating overinjection. We, therefore, have chosen to bring down $\eta$ and also the number of particles per cell for the monoenergetic injection case to $2 \times 10^{-3}$ and 4 , respectively. A comparison between the two energy spectra in the case of two injections is shown in Figure 12. In the case of Maxwellian injection, particles are seen to have gained energy up to $500 E_{\mathrm{sh}}$ (equivalent to $10 \mathrm{MeV}$ ), whereas for the monoenergetic injection recipe particles have gained energy up to $1000 E_{\mathrm{sh}}$ (equivalent to $20 \mathrm{MeV}$ ). The possible reason behind this discrepancy is that the particles have wide energy distribution in Maxwellian injection. Lower energy particles in the distribution have low probability of participating in the energization process. On the other hand, in monoenergetic injection, all particles being already suprathermal, the entire population may participate in the acceleration process and thus may end up with a higher energy tail in the energy spectrum.

\subsection{Quasi-perpendicular Shock}

It is well known that the particle acceleration mechanism in quasi-perpendicular shocks is much different than the parallel shocks. We, therefore, simulate a quasi-perpendicular shock with the same Alfvénic Mach number $(\approx 19)$. This time, the initial magnetic field makes an angle $\psi=75^{\circ}$ with the shock normal direction, making the shock quasi-perpendicular. The corresponding magnetic field components are now $B_{x}=B_{\mathrm{bg}} \cos (\psi)$ and $B_{y}=B_{\mathrm{bg}} \sin (\psi)$ with $B_{\mathrm{bg}}=3.55 B_{0}$ as mentioned in Section 3.1. Initially particles are injected following the same prescription mentioned in Section 2.2. Figure 13 shows the particle energy spectrum at the end of the simulation. It shows that the particles only get energized up to $40-45 E_{\text {sh. }}$.

Earlier hybrid-PIC (Caprioli \& Spitkovsky 2014a) simulation has shown a decrease in acceleration efficiency for quasiperpendicular shock. Naturally, this suggests the injection of a smaller number of suprathermal particles in the present case. We ran our quasi-perpendicular simulation for two more combinations of $\eta$ and the number of protons per cell, keeping the mass density of individual protons the same as our previous injection. The two sets include $\eta=1.5 \times 10^{-3}$ and $6 \times 10^{-4}$ with five and two protons per cell, respectively. The result is compared with our previous simulation where we considered $\eta=3 \times 10^{-3}$ and 10 protons per cell. Our new choices ensure a smaller amount of suprathermal particles in the domain. Though the resultant energy spectra from all three combinations vary in terms of the total number of protons, all of them show a very similar energization (Figure 14), indicating the fact that the population of injected suprathermal protons does not affect the overall energization process in quasi-perpendicular shock. The following analyses are performed on the simulation done with the injection prescribed in Section 2.2.

To understand the energization process, we need to understand which velocity component gains energy. Figure 15 depicts 2D particle velocity distribution of the downstream region. Both the velocity distribution functions show anisotropic nature. Figure 15 (a) shows that the velocity distribution $V_{x}-V_{y}$ is more skewed along an axis making $\approx 4^{\circ}$ with the $V_{x}$ axis, while $V_{y}-V_{z}$ is more skewed along the $V_{z}$ axis. This indicates particles are getting accelerated on a plane making an angle $\approx 4^{\circ}$ with the $X-Z$ plane.

We further investigated the mean magnetic field direction of the downstream region of interest (ROI) during the simulation's saturation phase. Here we remind the reader that this is a $2.5 \mathrm{D}$ simulation. Therefore, even though computation is performed only on a 2D plane, all three components of a vector field are evolved in the simulation. As our simulation is performed in the $\mathrm{X}-\mathrm{Y}$ plane, we assume that the local magnetic field $\boldsymbol{B}$ makes an angle $\theta$ with the $Y$-axis. To accommodate the third component of the magnetic field, we also assume $\phi$ to be the angle between $\boldsymbol{B}$ 's projection onto the $\mathrm{X}-\mathrm{Z}$ plane and the $\mathrm{Y}-\mathrm{Z}$ plane. We derive the value of $\theta$ and $\phi$ values at every grid of the ROI and the respective histograms are plotted to see the overall orientation of the field. Figures 16(a)-(b) demonstrate that in the ROI $\phi \approx 90^{\circ}$ is the dominant angle, which implies the mean $\boldsymbol{B}$ lies on the $\mathrm{X}-\mathrm{Y}$ plane. Figures $16(\mathrm{c})-(\mathrm{d})$ demonstrate that in the same downstream region $\theta \approx 4^{\circ}$ is the 


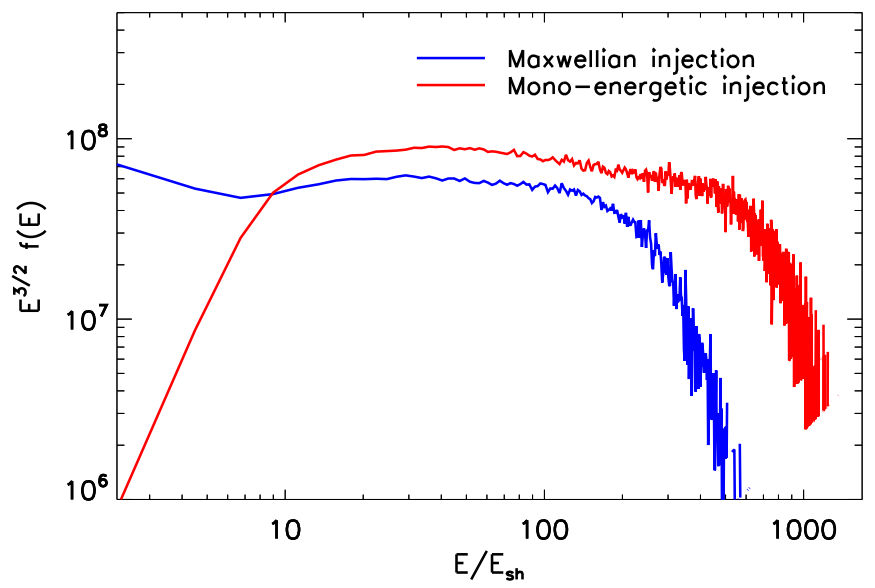

Figure 12. Saturated particle energy spectra for monoenergetic $\left(E_{\mathrm{inj}}=10 \mathrm{E}_{\mathrm{sh}}\right)$ and Maxwellian injection.

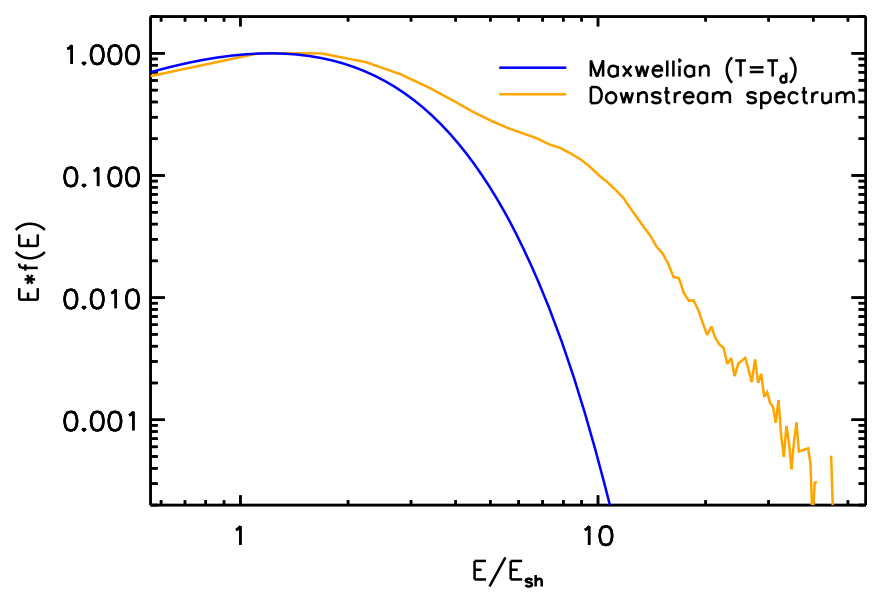

Figure 13. Injected Maxwellian and saturated energy spectra for quasiperpendicular shock with Mach number $\approx 19$.

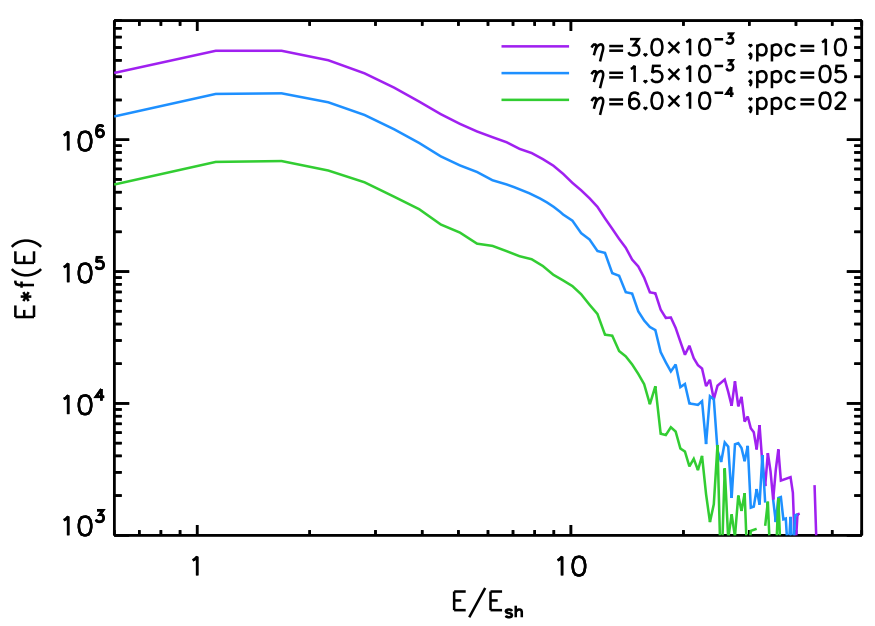

Figure 14. Energy spectra derived from the simulation of quasi-perpendicular shock with $M_{A} \approx 19$, by changing injection fraction $(\eta)$ and particles per cell (ppc).

dominant angle. Overall Figure 16 concludes that the mean $\boldsymbol{B}$ lies in the $\mathrm{X}-\mathrm{Y}$ plane and makes an angle $\approx 4^{\circ}$ with the $Y$-axis.

After knowing the accelerated components of the particles' velocity and the orientation of the mean magnetic field in the ROI, it is now understood that particles are predominantly getting accelerated in the plane perpendicular to the mean magnetic field (i.e., the plane making an angle $\approx 4^{\circ}$ with the $X-$ $\mathrm{Z}$ plane). The convective electric field $(\boldsymbol{E}=-\boldsymbol{u} \times \boldsymbol{B})$ is the reason behind this. The field acts on the plane perpendicular to the mean magnetic field and accelerates protons on the same plane. Concurrently, the local grad B force, developed across the shock front due to shock compression, makes the particles drift on the plane of the shock. Additionally, the particles' velocity components perpendicular to the mean magnetic field, namely the $V_{\perp}$ component, increases in magnitude. The parallel component $V_{\|}$still maintains the injected distribution, resulting in an anisotropic 2D velocity distribution behind the shock. The mechanism is popularly known as SDA and plays a major role in accelerating protons in our quasi-perpendicular shock simulation.

We should point out that although the Alfvénic Mach of both the parallel and quasi-perpendicular shocks are the same in our abovementioned simulations, the energy gained by the protons in the quasi-perpendicular shock case is significantly less. This can be explained with Figure 17. Only a few high-energy protons are seen to have escaped the shock front and move into the upstream region. After getting accelerated by SDA, protons are mostly advected into the downstream region within a few gyrocycles, resulting in the truncation of further energization process. The few high-energy protons that managed to escape the shock front are insufficient to generate strong fluctuations in the upstream region. Nearly nonfluctuating density $\left(\delta \rho / \rho_{0} \approx 0\right)$ in the upstream implies that there is no active participation of DSA in our simulation to accelerate protons. However, in reality, inherent fluctuations of the SW can excite DSA and may accelerate particles further. This will be investigated in our future work.

\section{ICME Simulation for Low Mach Shock}

ICME shocks with low Alfvénic Mach numbers (Berdichevsky et al. 2000; Oh et al. 2007; Lugaz et al. 2015) are more common. Keeping that in mind, we also have simulated ICME shocks with low Alfvénic Mach $\approx 10$ and 4 . Generation of shock and particle injection recipes are identical to the cases discussed in Section 3. The only parameter that is changed in order to produce shocks with low Machs is the relative velocity between the SW and ICME plasma. For Alfvénic Mach $\approx 10$, we have injected a SW flow with relative speed $-50.05 v_{A}$ $\left(=976 \mathrm{~km} \mathrm{~s}^{-1}\right)$, whereas for Alfvénic Mach $\approx 4$ the relative speed is $-20 v_{A}\left(=-390 \mathrm{~km} \mathrm{~s}^{-1}\right)$. Both these shocks are parallel in nature.

Figure 18 exhibits the particle energy spectrum for the $M_{A} \approx 10$ shock. Particles are seen to get energized up to $300 E_{\mathrm{sh}}$ in this simulation. The flat part of the spectrum directs toward the DSA mechanism for particle acceleration. On the other hand, Figure 19, which depicts the particle energy spectrum for the shock with Alfvénic Mach $\approx 4$, shows no indication of particle acceleration.

Therefore, these sets of numerical simulations follow our intuitive guess that Alfvénic Mach number is a good indicator of shocks' capability to accelerate particles. Low Mach shocks may not work as good accelerators.

\section{Summary and Discussion}

This paper has investigated the particle acceleration process in ICME shocks. We used the MHD-PIC module of the PLUTO code (Mignone et al. 2018). The neutral background 
(a)

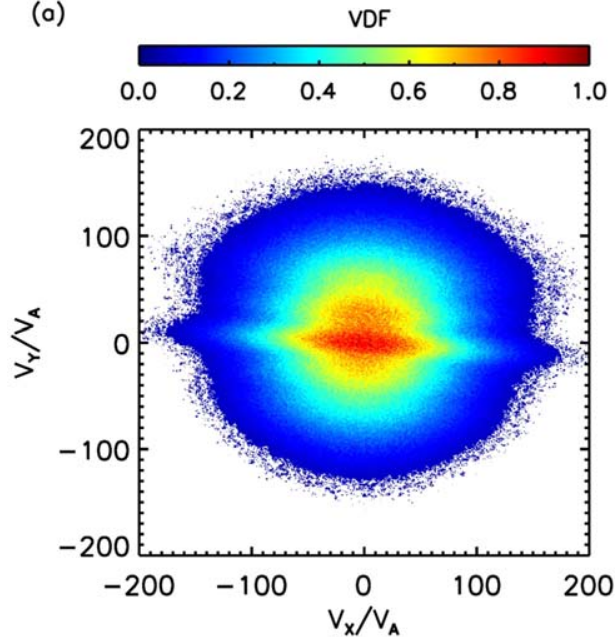

(b)
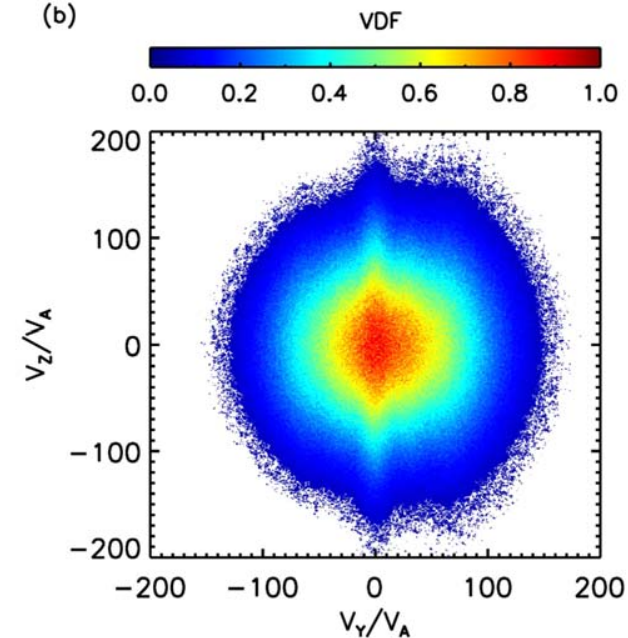

Figure 15. 2D velocity distribution function of the quasi-perpendicular shock with $M_{A} \approx 19$. Particles were collected from just behind the shock. The region is marked in Figure 16.
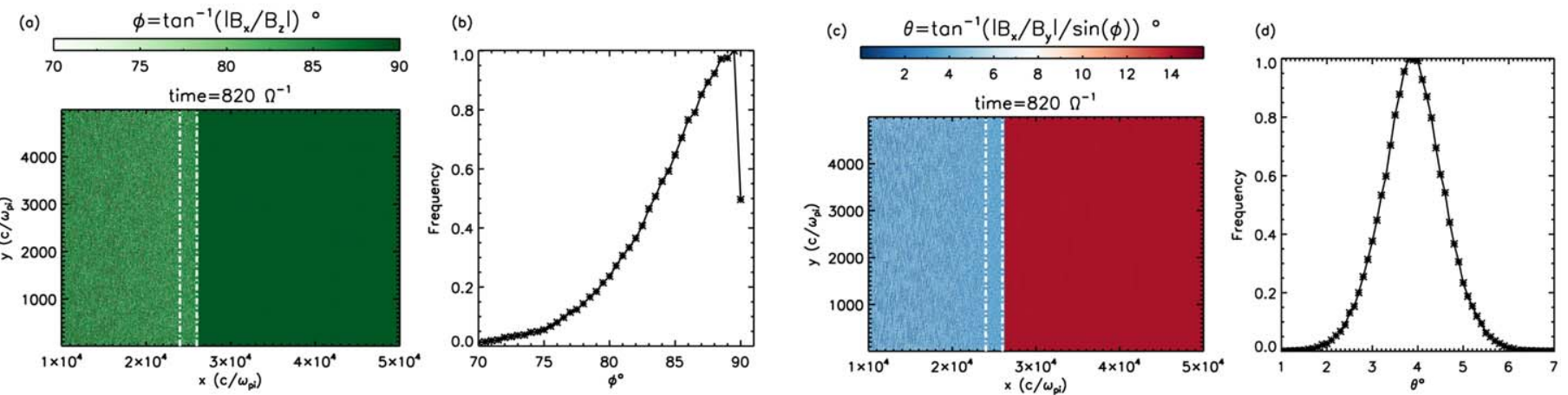

Figure 16. The orientation of the magnetic field just behind the shock. The particular region of interest is marked with vertical dashed lines. (a) Contour plot of the angles $(\phi)$ between the Y-Z plane and the projection of $\boldsymbol{B}$ onto the X-Z plane. (b) A histogram depicting the dominant $\phi$ angle of the region of interest, showing that the mean magnetic field of the region lies on the $\mathrm{X}-\mathrm{Y}$ plane. (c) Contour plot of the angle between $\boldsymbol{B}$ and the $Y$-axis. (d) Histogram plot of the region of interest to show the dominant $\theta$ angle showing the mean field makes an angle $\approx 4^{\circ}$ with the $Y$-axis. Particle velocity distribution function of the same region is plotted in Figure 15.
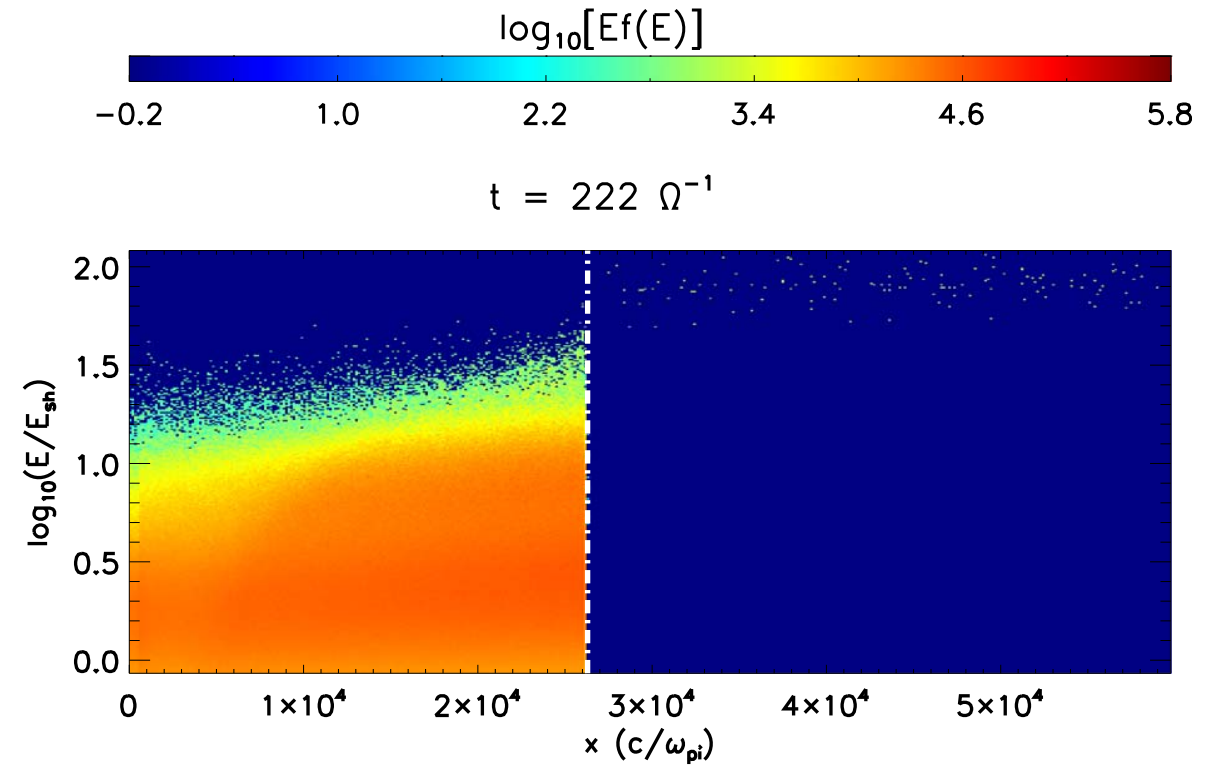

Figure 17. 2D energy spectrum, showing the particle energy distribution as a function of position $x$. Here $E_{\mathrm{sh}}$ is the shock energy given by $E_{\mathrm{sh}}=v_{u}^{2} / 2$ where $v_{u}$ is the upstream plasma speed in the downstream reference frame. Location of the shock front is indicated by the white dashed line. As evident from this plot, high-energy particles outrunning the shock are very few in number and hence cannot seed strong fluctuations in the upstream. 


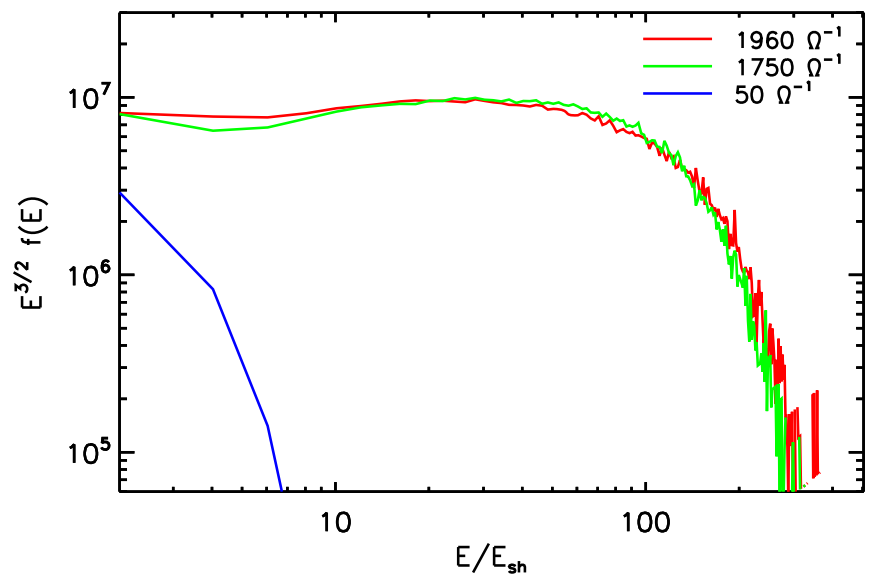

Figure 18. Evolution of downstream spectra for a parallel shock with $M_{A} \approx 10$, calculated over a region of width $1000\left(c / \omega_{\text {pi }}\right)$ just behind the shock.

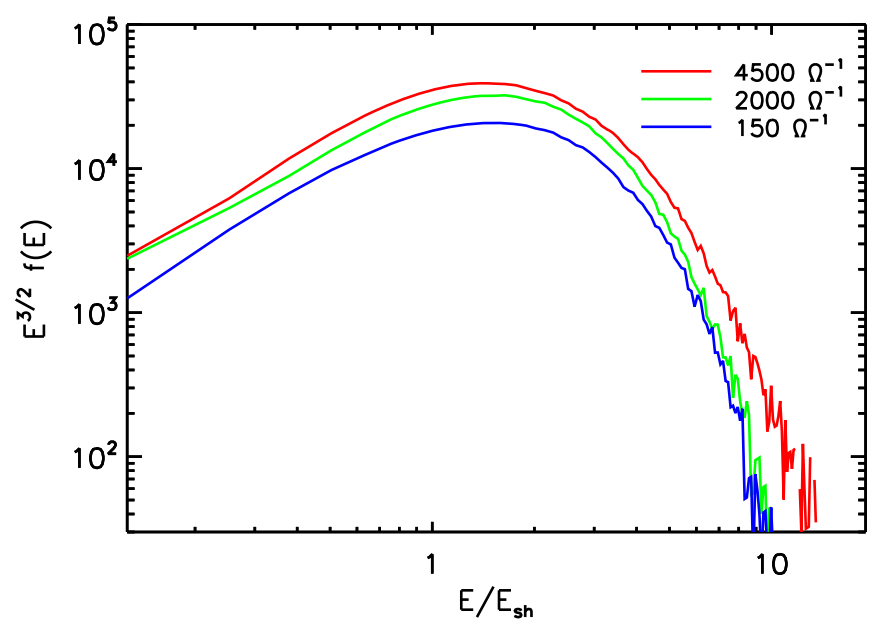

Figure 19. Evolution of downstream spectra for a parallel shock with $M_{A} \approx 4$, calculated over a region of width $1000\left(c / \omega_{\text {pi }}\right)$ just behind the shock.

plasma is treated as a magnetohydrodynamic fluid, and nonthermal proton dynamics is taken care of using a PIC code. The reactions of particle dynamics onto the MHD fluid and vice versa are appropriately managed. Compared to earlier heliospheric shock models (Zank et al. 2000), this model is more local in nature. Unlike the former, it can only simulate a small region across the shock. But, particles evolve selfconsistently on the background thermal plasma. This hybrid model is more similar to Gargaté et al.'s (2014) model, but computationally much less expensive. Such a hybrid simulation enables us to follow the particle dynamics and fluid evolution until the particle energy spectrum nearly saturates. These simulations empower us to predict various in situ observations and help us plan future missions.

We started our investigation with a $\mathrm{CME}$ shock having an extremely high Alfvénic Mach number $\left(M_{A} \approx 19\right)$. Even though such a strong shock is rare in the heliosphere, one was evidenced lately (on 2012 July 23). The particle acceleration process has been probed initially for a parallel shock, where the shock propagation direction exactly matches with the ambient SW magnetic field. This being an idealistic case, we also have studied a quasi-perpendicular shock, where the ambient magnetic field forms a $75^{\circ}$ angle with the propagation direction. CME shocks with lower Mach numbers (e.g., $M_{A} \approx 10$ and 4 ) have also been investigated in the present study. Particles are injected at the shock front in all our simulations. During injection, the particles' distribution follows a Maxwellian with characteristic downstream temperature (i.e., energy $\left.E_{\mathrm{sh}}\right)$. In the following, we outline our results as well as suggest ways to further explore the observation data that may confirm various physical processes occurring around such CME shocks.

Instability and dynamo in the parallel shock-The first simulation, which delineates a plane parallel shock with high Alfvénic Mach $\left(M_{A} \approx 19\right)$, demonstrates that such a shock can energize protons up to $500 E_{\mathrm{sh}}(\approx 10 \mathrm{MeV})$. The slope of the particle energy spectrum (Figure 10) indicates the role of DSA in the particle acceleration process. We also note that once particles get energized, many of them leave the shock front and move to the far upstream region (Figure 8).

The upstream of the shock is seen to become turbulent with the growth of density cavities. Such cavities are due to the development of nonresonant Bell instability in the upstream region. As the instability grows, the sizes of these cavities increase. Once the local ion-gyroradius becomes comparable to the cavity size, the instability ceases to grow (Figure 2). We have further investigated the existence of resonant streaming instability in the domain. We find that the longest and shortest resonant modes encompass the peak of the magnetic power spectrum. However, the fastest growing mode of nonresonant Bell instability turns out to be responsible for the peak of the power spectrum of the region's transverse magnetic field (Figure 4).

The turbulent magnetic field of the shock downstream region is seen to have enhanced more than is expected from the shock compression, hinting at the presence of a dynamo mechanism in the region. Due to nonaligned pressure and density gradient at the corrugated shock front, instability like RichtmyerMeshkov instability may kick in and develop turbulence. Such turbulence stretches the field lines and thereby enhances the local magnetic field. Kinetic or magnetic diffusivity are not explicitly specified in our simulation. Nevertheless, when we compare the kinetic and magnetic energy spectra (Figure 7) the dominant magnetic energy toward the small scale $\left(k>0.07\left(c / \omega_{\mathrm{pi}}\right)^{-1}\right)$ indicates the development of small scale dynamo in the region.

Quasi-perpendicular shock demonstrates velocity anisotropy -We have also performed simulation of quasi-perpendicular shock with a high Alfvénic Mach number $\left(M_{A} \approx 19\right)$. The particle energy spectrum shown in Figure 13 indicates energization up to $40-45 E_{\mathrm{sh}}$, which is much smaller than the particle energization in the parallel shock with the same Alfvénic Mach. In quasi-perpendicular shock, the particle energization is expected due to the SDA process, where the convective electric field $\boldsymbol{E}=-\boldsymbol{u} \times \boldsymbol{B}$ plays a vital role in accelerating the particles. Activation of SDA in the present case is evident from the particles' velocity distribution as shown in Figure 15. The distribution shows the development of anisotropy on the plane perpendicular to the local mean magnetic field.

Our simulations demonstrate that, in quasi-perpendicular shocks (Figure 17), high energetic particles rarely escape to the upstream and therefore do not develop strong turbulence in the region, as it happens in the parallel shock. This also prevents the particles from participating in DSA and accelerating further. However, once again, the SW turbulence may play a crucial 
role in accelerating these particles, which is not incorporated in the present simulations.

Low Mach shocks are less effective to accelerate particlesAs expected, our simulations of low Mach number shocks show no effective particle acceleration. We have simulated parallel shocks with Alfvénic Mach, $M_{A} \approx 10$ and 4. Though the shock with $M_{A} \approx 10$ could energize particles up to $300 E_{\mathrm{sh}}$, the shock with $M_{A} \approx 4$ does not show evidence of particle acceleration.

It is worth mentioning that our simulations were unable to produce particles with very high energy (e.g., up to $100 \mathrm{MeV}$, as in the case of real observations Russell et al. 2013). Multiple reasons can be responsible for this. While traveling from the Sun to $1 \mathrm{au}$, the CME shocks encounter variable backgrounds over a long duration of time. This may help particles to get energized continuously. On the other hand, our localized simulations are provided with a background atmosphere of 1 $\mathrm{au}$, and therefore can only energize particles to whatever energies are possible over this background. Furthermore, our present simulations start with a uniform SW background. The inherent fluctuations of the real SW may energize particles to a greater extent, which needs to be investigated in the future. The restricted input energy distribution of the particles may also be responsible for the limited energization process. In the simulations, we inject particles with a Maxwellian distribution having a characteristic temperature of the downstream. However, these may not be the only particles getting induced at the shock front in reality. Some amount of much higher energetic particles that are already energized due to magnetic reconnection may also get induced at the shock front for further processing and may give rise to the observed high-energy tails. Such a possibility has been proposed earlier(Klein \& Trottet 2001). As others (e.g., Bai et al. 2015; Mignone et al. 2018), we also have attempted to inject particles with $10 E_{\mathrm{sh}}$. The resultant energy distribution gets extended toward the higher end but only by a few MeVs as compared to the Maxwellian injection (Figure 12). We have also shown that the limited size of the simulation domain may restrict the growth of more extended modes in the domain and regulate energy growth beyond a threshold.

Understanding particle acceleration in shocks is a major topic in overall astrophysics. Shocks of different strengths and magnetic configurations are ubiquitous in the heliosphere. Heliospheric shocks can be quasi-parallel or quasi-perpendicular, depending on the relative angle between their propagation direction and ambient upstream magnetic field. Therefore, shocks that are potential accelerators (relatively high $M_{A}$ ) should be able to demonstrate the characteristics of both DSA and SDA mechanisms. The issue of particle acceleration, therefore, can be explored directly by using heliospheric in situ data. In the following paragraph, we highlight a few observables that can confirm our current understanding of the subject.

In principle, SEP energy spectra should be able to demonstrate the characteristic $-3 / 2$ slope if DSA is the dominant mechanism. On the other hand, the $2 \mathrm{D}$ velocity distribution of the particles should be able to demonstrate anisotropy if SDA plays a pivotal role in accelerating particles. The existence of nonresonant Bell instability can be verified by measuring the size of the upstream density cavities or by analyzing the upstream magnetic turbulence. It will also be interesting to see if the downstream magnetic field gets enhanced due to turbulent dynamo.

It is possible to perform local numerical simulations of wide ranges of ICME shocks and compare the simulation outputs with real observations. ICME shocks with varied strength at different heliospheric distances can be compared with the simulation results. We can also corroborate the effect of different SW background plasma on ICME shocks. We envisage data from the Integrated Science Investigation of the Sun (IS $\odot$ IS) and Electromagnetic Fields Investigation experiments on board the Parker Solar Probe, the Energetic Particle Detector, Solar Wind Plasma Analyser and Magnetometer on board Solar Orbiter or the Aditya Solarwind Particle EXperiment on board Indian Space Research Organisation's AdityaL1 mission will be able to probe in this direction.

Computations were carried out on the Physical Research Laboratory's VIKRAM cluster. We thank the anonymous referee for insightful comments, which helped us improve the manuscript. B.V. is thankful for the support provided by the Max Planck Partner Group established at IIT Indore.

\section{ORCID iDs}

Shanwlee Sow Mondal (D) https://orcid.org/0000-00034225-8520

Aveek Sarkar (1) https://orcid.org/0000-0002-4781-5798

Bhargav Vaidya (iD https://orcid.org/0000-0001-5424-0059

Andrea Mignone (D) https://orcid.org/0000-0002-8352-6635

\section{References}

Amato, E., \& Blasi, P. 2009, MNRAS, 392, 1591

Armstrong, T. P., Pesses, M. E., \& Decker, R. B. 1985, GMS, 35, 271

Bai, X.-N., Caprioli, D., Sironi, L., \& Spitkovsky, A. 2015, ApJ, 809, 55

Baring, M. G., Ogilvie, W., Ellison, C., \& Forsyth, R. J. 1997, ApJ, 476, 889

Bell, A. R. 1978, MNRAS, 182, 147

Bell, A. R. 2004, MNRAS, 353, 550

Bell, A. R. 2005, MNRAS, 358, 181

Berdichevsky, D. B., Szabo, A., Lepping, R. P., Viñas, A. F., \& Mariani, F. 2000, JGRA, 105, 27289

Blandford, R. D., \& Ostriker, J. P. 1978, ApJL, 221, L29

Brouillette, M. 2002, AnRFM, 34, 445

Caprioli, D., \& Spitkovsky, A. 2013, ApJL, 765, L20

Caprioli, D., \& Spitkovsky, A. 2014a, ApJ, 783, 91

Caprioli, D., \& Spitkovsky, A. 2014b, ApJ, 794, 46

Caprioli, D., \& Spitkovsky, A. 2014c, ApJ, 794, 47

Decker, R. B. 1983, JGR, 88, 9959

Decker, R. B. 1988, SSRv, 48, 195

Desai, M., \& Giacalone, J. 2016, LRSP, 13, 3

Fermi, E. 1949, PhRv, 75, 1169

Fermi, E. 1954, ApJ, 119, 1

Feynman, J., \& Gabriel, S. B. 2000, JGR, 105, 10543

Gargaté, L., Bingham, R., Fonseca, R. A., \& Silva, L. O. 2007, CoPhC, 176,419

Gargaté, L., Fonseca, R. A., Silva, L. O., Bamford, R. A., \& Bingham, R. 2014, ApJ, 792, 9

Giacalone, J., \& Jokipii, J. R. 2007, ApJL, 663, L41

Gopalswamy, N. 2003, AdSpR, 31, 869

Helder, E. A., Vink, J., Bykov, A. M., et al. 2012, SSRv, 173, 369

Hu, J., Li, G., Ao, X., Zank, G. P., \& Verkhoglyadova, O. 2017, JGRA, 122, 10,938

Kahler, S. W. 2001, JGRA, 106, 20947

Kahler, S. W. 2004, ApJ, 603, 330

Kahler, S. W., \& Vourlidas, A. 2005, JGRA, 110, A12S01

Kahler, S. W., \& Vourlidas, A. 2013, ApJ, 769, 143

Kilpua, E., Koskinen, H. E. J., \& Pulkkinen, T. I. 2017, LRSP, 14, 5

Klein, K.-L., \& Dalla, S. 2017, SSRv, 212, 1107

Klein, K.-L., \& Trottet, G. 2001, SSRv, 95, 215

Kouloumvakos, A., Rouillard, A. P., Wu, Y., et al. 2019, ApJ, 876, 80 
Krymskii, G. F. 1977, DoSSR, 234, 1306

Lee, M. A., \& Fisk, L. A. 1982, SSRv, 32, 205

Li, G., Shalchi, A., Ao, X., Zank, G., \& Verkhoglyadova, O. P. 2012, AdSpR, 49, 1067

Li, G., Zank, G. P., Desai, M. I., Mason, G. M., \& Rice, W. K. M. 2005a, GMS, 156, 51

Li, G., Zank, G. P., \& Rice, W. K. M. 2003, JGRA, 108, 1082

Li, G., Zank, G. P., \& Rice, W. K. M. 2005b, JGRA, 110, A06104

Lipatov, A. S. 2002, The Hybrid Multiscale Simulation Technology: An Introduction with Application to Astrophysical and Laboratory Plasmas (New York: Springer)

Lugaz, N., Farrugia, C. J., Smith, C. W., \& Paulson, K. 2015, JGRA, 120, 2409

Mignone, A., Bodo, G., Vaidya, B., \& Mattia, G. 2018, ApJ, 859, 13

Mizuno, Y., Pohl, M., Niemiec, J., et al. 2011, ApJ, 726, 62

Oh, S. Y., Yi, Y., \& Kim, Y. H. 2007, SoPh, 245, 391

Podesta, J. J., Roberts, D. A., \& Goldstein, M. L. 2007, ApJ, 664, 543

Reames, D. V. 1999, SSRv, 90, 413

Reames, D. V. 2002, ApJL, 571, L63

Reames, D. V. 2012, ApJ, 757, 93

Reville, B., \& Bell, A. R. 2012, MNRAS, 419, 2433
Rice, W. K. M., Zank, G. P., \& Li, G. 2003, JGRA, 108, 1369

Riley, P., Caplan, R. M., Giacalone, J., Lario, D., \& Liu, Y. 2016, ApJ, 819, 57

Romero, G. E., Boettcher, M., Markoff, S., \& Tavecchio, F. 2017, SSRv, 207, 5

Russell, C. T., Mewaldt, R. A., Luhmann, J. G., et al. 2013, ApJ, 770, 38

Ryan, J. M., Lockwood, J. A., \& Debrunner, H. 2000, SSRv, 93, 35

Sano, T., Nishihara, K., Matsuoka, C., \& Inoue, T. 2012, ApJ, 758, 126

Sheeley, N. R., Howard, J., \& Koomen, R. A. 1983, NASA Conf. Publication, Vol. 228 (Washington, DC: NASA), 693

Spitkovsky, A. 2005, in AIP Conf. Ser. 801, Astrophysical Sources of High Energy Particles and Radiation, ed. T. Bulik, B. Rudak, \& G. Madejski (Melville, NY: AIP), 345

van Marle, A. J., Casse, F., \& Marcowith, A. 2018, MNRAS, 473, 3394

Verkhoglyadova, O. P., Li, G., Zank, G. P., et al. 2010, JGRA, 115, A12103 Verkhoglyadova, O. P., Li, G., Zank, G. P., Hu, Q., \& Mewaldt, R. A. 2009, ApJ, 693, 894

Zank, G. P., Li, G., Florinski, V., et al. 2004, JGRA, 109, A04107 Zank, G. P., Li, G., Florinski, V., et al. 2006, JGRA, 111, A06108

Zank, G. P., Li, G., \& Verkhoglyadova, O. 2007, SSRv, 130, 255

Zank, G. P., Rice, W. K. M., \& Wu, C. C. 2000, JGR, 105, 25079 